\title{
Mirella D'ASCENZO, Tra centro e periferia. La Scuola elementare a Bologna dalla Daneo-Credaro all'avocazione statale (1911-1933)
}

Bologna, Cooperativa Libraria Universitaria Editrice, 2006, 439 p.

Michel Ostenc

\section{QpenEdition}

\section{Journals}

Édition électronique

URL : http://journals.openedition.org/assr/21114

DOI : 10.4000/assr.21114

ISSN : $1777-5825$

Éditeur

Éditions de l'EHESS

Édition imprimée

Date de publication : 31 décembre 2009

Pagination : $75-342$

ISBN : 978-2-7132-2218-4

ISSN : 0335-5985

\section{Référence électronique}

Michel Ostenc, « Mirella D'AscEnzo, Tra centro e periferia. La Scuola elementare a Bologna dalla DaneoCredaro all'avocazione statale (1911-1933) », Archives de sciences sociales des religions [En ligne], 148 octobre-décembre 2009, document 148-39, mis en ligne le 19 mai 2009, consulté le 21 septembre 2020. URL : http://journals.openedition.org/assr/21114 ; DOI : https://doi.org/10.4000/assr.21114

Ce document a été généré automatiquement le 21 septembre 2020.

(C) Archives de sciences sociales des religions 


\section{Mirella D'ASCENZO, Tra centro e periferia. La Scuola elementare a Bologna dalla Daneo-Credaro all'avocazione statale (1911-1933)}

Bologna, Cooperativa Libraria Universitaria Editrice, 2006, 439 p.

\section{Michel Ostenc}

\section{RÉFÉRENCE}

Mirella D'ASCENZO, Tra centro e periferia. La Scuola elementare a Bologna dalla DaneoCredaro all'avocazione statale (1911-1933), Bologna, Cooperativa Libraria Universitaria Editrice, 2006, $439 \mathrm{p}$.

1 L'ouvrage de Mirella D'Ascenzo utilise l'abondante documentation d'archives de Bologne pour étudier la politique scolaire des municipalités successives de la ville à partir de 1911, le rôle des associations d'enseignants dans les luttes revendicatives de la profession et dans la création d'activités parascolaires qui accompagnèrent le passage de l'école publique sous l'autorité de l'État; mais le livre n'est pas seulement une monographie d'histoire locale et il s'insère dans un cadre législatif national s'inspirant du profond renouveau de la culture pédagogique italienne entre l'époque de Giolitti et celle du fascisme.

2 La loi Orlando du 8 juillet 1904 s'efforçait de lutter contre l'analphabétisme en prolongeant l'obligation scolaire jusqu'à douze ans. Elle avait obtenu le soutien politique de la gauche qui y voyait des possibilités de développements ultérieurs, mais son application se heurta à d'insurmontables difficultés. Certes, la loi créait une assistance scolaire aux familles financée par l'État sous forme de subventions aux communes; mais beaucoup d'entre elles n'avaient pas les moyens d'ouvrir trois nouvelles classes dans leurs écoles et le bilan s'avéra décevant. En 1908, la moitié 
seulement des élèves inscrits étaient reçus à l'examen de la $\mathrm{III}^{\mathrm{e}}$ classe, à peine le tiers poursuivait leurs études dans la $\mathrm{IV}^{\mathrm{e}}$ et treize mille obtenaient une licence à la fin de la $\mathrm{VI}^{e}$, chiffres dérisoires pour quatre millions et demi d'enfants d'âge scolaire. La loi Sonnino du 15 juillet 1906 prévoyait une aide publique aux écoles ambulantes destinées à scolariser les enfants des zones déficientes du Midi ; mais il n'existait que trois mille établissements de ce type en 1908 sur un total de soixante-trois mille. L'enquête du Directeur général de l'Instruction publique Camillo Corradini montrait clairement le lien existant entre l'école et la question sociale. La gratuité ne suffisait pas à satisfaire l'obligation et il fallait y ajouter l'assistance. De retour au pouvoir en 1909, Sonnino avait choisi Edoardo Daneo comme ministre de l'Instruction publique. C'était un laïc modéré qui souhaitait lutter contre l'analphabétisme en étatisant les écoles des petites communes, tout en respectant l'autonomie des plus grandes. Son projet échoua, mais il fut repris avec succès par Luigi Credaro en 1911. La loi Daneo-Credaro l'emporta grâce au soutien politique de Sonnino, des radicaux et des socialistes.

3 Sans altérer l'organisation scolaire de la loi Casati de 1859, la loi Daneo-Credaro introduisait le double régime administratif des écoles d'Etat et des écoles communales. Elle donnait satisfaction aux communes méridionales favorables à l'étatisation, et à celles du nord qui refusaient d'abandonner la gestion de leurs écoles. Seules les communes trop pauvres pour continuer à y faire face cédaient à la province l'administration de leurs écoles primaires, l'État se chargeant du financement; mais elles étaient contraintes, lorsque leur établissement ne comptait que trois classes, d'en ouvrir une quatrième avec horaire alterné. Les chefs-lieux de provinces et de cercles conservaient ainsi la direction et la gestion de leurs écoles ; mais en 1922, elles n'étaient plus que deux cent soixante quatre, pendant que plus de huit mille communes avaient abandonné leur école à des conseils scolaires provinciaux. La loi Daneo-Credaro donnait de la stabilité à la carrière des instituteurs, tout en mécontentant leur principale association l'Unione Magistrale Nazionale (UMN) qui aurait souhaité une étatisation totale. Le catholicisme conservateur, très attaché à la liberté de l'enseignement et à l'instruction religieuse à l'école, était hostile à l'étatisation; mais les associations d'enseignants catholiques, et notamment la «Niccolò Tommaseo » née en 1906, se montraient plus conciliantes malgré leur crainte d'une atteinte à l'autonomie communale. La loi permettait en effet aux catholiques de siéger dans les conseils provinciaux. L'étatisation des écoles provoqua une augmentation massive des charges des finances publiques sans soulager pour autant les finances locales. Le problème ne sera résolu qu'en 1931, avec l'étatisation définitive de toutes les écoles primaires du pays.

4 La municipalité conservatrice de Bologne fut confrontée dès 1904 à la question du traitement des maîtres et à leur égalité avec celui des institutrices, dans une profession de plus en plus féminisée. Un règlement organique fut institué qui distinguait le cas des écoles urbaines de celui des zones rurales. Toutefois, l'extension de l'obligation scolaire, la création d'un enseignement primaire long au cours populaire et une demande croissante d'enseignement se heurtèrent à l'insuffisance des locaux. Pour y faire face, la municipalité dut revenir sur les dispositions du Règlement et elle entra en conflit avec les enseignants. En outre, les instituteurs de Bologne souhaitaient utiliser les Cours populaires à des fins de formation professionnelle, alors que la municipalité voulait leur conserver une vocation d'enseignement général. Les diverses tendances politiques de la ville s'exprimaient dans de grands organes de presse. "Il Resto del Carlino» défendait le projet équilibré de Daneo et «Il Giornale del Mattino» des 
libéraux démocrates préférait celui de Credaro. Le quotidien catholique "L'Avvenire d'Italia » s'inquiétait par contre des alliances nouées par Sonnino avec les démocrates anticléricaux et faisait preuve d'une grande méfiance à l'égard de Credaro. Le journal défendait les prérogatives communales qu'il considérait comme le prolongement naturel des droits de la famille; mais cette tendance du catholicisme s'opposait à la Lega Democratica Nazionale, fondée par Romolo Murri à Bologne en 1905, qui préconisait la suppression de l'instruction religieuse dans les écoles publiques et l'intervention de l'État dans les cas avérés d'incapacité financière des communes. Le sénateur de Bologne Alberto Dallolio soutint la loi tout en émettant quelques réserves sur son application. Il s'interrogeait sur le rôle du provéditeur dont les pouvoirs étaient réduits par la création d'organismes chargés de la gestion des écoles de la province. Il s'agissait d'une Délégation gouvernementale assumant le contrôle financier sous l'autorité du préfet et du Conseil scolaire, cheville ouvrière de la nouvelle organisation, qui comprenait des représentants élus des enseignants et de l'ensemble de la population. Le sénateur réclamait par ailleurs une réforme des écoles normales, afin d'améliorer la formation professionnelle des maîtres, et il s'inquiétait de la situation des communes autonomes qui nécessitait une réforme urgente des finances locales. L'Unione Magistrale Nazionale disposait d'une importante représentation à Bologne où s'était tenu en mars 1902 l'un de ses premiers congrès. La ligne apolitique symbolisée par le mot d'ordre « Ni esclaves, ni rebelles " y avait été solennellement proclamée. La composition des organes dirigeants de la section de Bologne va rester presque inchangée jusqu'en 1924. Elle soutenait le projet de Daneo qui évitait l'étatisation des grandes communes, mais reprochait son caractère bureaucratique au Conseil scolaire de la loi Credaro. Il existait pourtant à l'intérieur de la Fédération bolonaise un courant proche du syndicalisme révolutionnaire qui s'exprimait dans le périodique «La Scuola moderna ». Ces anarchistes entendaient refondre la société au moyen d'une école rationaliste et anticléricale dont l'éducation intégrale respecterait les droits de l'enfant.

L'application de la loi Daneo-Credaro s'éternisait et elle décevait ses plus chauds partisans. Son échec était attribué à la paralysie d'un Conseil scolaire enlisé dans les procédures de l'administration provinciale; mais l'organe national des instituteurs «I Diritti della Scuola» dénonçait régulièrement ses décisions qui répartissaient des faveurs ou assouvissaient des vengeances. La mairie de Bologne tardait à mettre en place des patronages scolaires qu'elle souhaitait confier à des associations privées. La législation durcissait les contraintes pesant sur les maitres suppléants et la loi Comandini du 21 juillet 1909 instaurait un système de concours qui établissait une hiérarchie plus stricte dans la profession. Si bien que le périodique bolonais de l'UMN «La Voce dei Maestri » défendait avec virulence les intérêts des enseignants. La situation scolaire s'améliorait, l'obligation étant respectée par plus de $90 \%$ des enfants de la province en 1912, alors que la moyenne nationale de l'analphabétisme était encore de $46,7 \%$.

6 L'auteur fait l'éloge de la municipalité socialiste qui a géré Bologne de 1912 à 1922. La ville mit d'abord l'accent sur la laïcité de ses écoles. Elle organisa au Cours populaire les enseignements professionnels que les associations magistrales avaient toujours réclamés et elle confia à l'Institut commercial de Bologne le soin de former les maîtres de ces disciplines. L'objectif était de permettre aux élèves sortis de l'école primaire de poursuivre leurs études jusqu'à quinze ans, avant d'être en âge d'entrer dans des écoles professionnelles spécialisées. La municipalité multiplia les instruments pédagogiques et les organismes parascolaires, créa des asiles pour accueillir la petite enfance et mit en 
place un réseau d'éducateurs dans les quartiers populaires de la ville. La recherche de nouveaux modèles éducatifs l'incita à s'inspirer d'une pédagogie proche de l'idéalisme de Giuseppe Lombardo Radice dans ses écoles de plein air. Cette politique scolaire fut couronnée de succès et le taux d'analphabétisme à Bologne tomba de 15 à $9 \%$ entre 1911 et 1922.

7 Les rapports se tendirent au sein de la classe enseignante dès la fin de la guerre. La présidence de l'UMN était assurée depuis 1916 par le républicain Ubaldo Comandini qui tenait au maintien du caractère apolitique de l'association et s'efforçait de lui éviter toute évolution vers des formes syndicales. La tentation était d'autant plus forte qu'en 1919 les instituteurs proches du maximalisme révolutionnaire avaient fondé le Syndicat magistral à Milan, et cette tendance se manifestait à Bologne au sein d'une "Lega insegnanti » particulièrement active. La scission devenait inévitable et la «vieille garde » de l'U.M.N. parvenait à regrouper la plupart des maîtres titulaires au sein d'une Fédération provinciale, alors que la «Lega» recrutait ses membres parmi les enseignants plus jeunes et chez les suppléants. Au lendemain de la victoire socialiste aux élections municipales de novembre 1922, Bologne fut administrée par un commissaire préfectoral qui revint progressivement sur les réalisations précédentes.

8 Le lent déclin de la «Lega insegnanti » de Bologne profita à la Fédération provinciale qui multiplia les attaques contre le Syndicat magistral et le «bolchévisme » local. La section bolonaise de la «Niccolò Tommaseo » faisait campagne pour la liberté de l'enseignement et la question de l'instruction religieuse était débattue favorablement dans un colloque d'avril 1923. Deux nouvelles organisations firent leur apparition en marge de ces luttes politiques et idéologiques. Le Groupe d'action pour les écoles primaires naquit à Bologne en décembre 1920, répondant à l'appel des pédagogues Ernesto Codignola et Giuseppe Lombardo Radice qui militaient pour un renouveau idéaliste de l'enseignement. La section locale de la Fédération des instituteurs anciens combattants, fondée par des nationalistes comme Balbino Giuliano, exigeait la titularisation de ses membres qui avaient été reçus à un concours de recrutement en 1919 et restaient dépourvus de postes. La réforme Gentile de 1923 supprima l'enseignement primaire long, les communes qui en avaient les moyens organisant des "Cours intégratifs » en trois ans pour prolonger la scolarité jusqu'à quatorze ans. La réforme modifia profondément la composition du corps enseignant, en contraignant les maîtres en fin de carrière à prendre leur retraite et en titularisant à leur place des instituteurs anciens combattants. Les programmes accordaient une place importante à la spontanéité de l'élève en considérant le dessin libre comme son meilleur mode d'expression. Ils s'inspiraient des innovations pédagogiques préconisées par l'Éducation nouvelle. Les enseignants de Bologne manifestèrent leur intérêt pour ce mode d'expression en organisant une exposition consacrée au langage graphique de l'enfant.

La fascisation de l'école s'effectua très progressivement, les instituteurs semblant se satisfaire d'un régime politique qui leur accordait des places importantes au sein de ses organisations de jeunesse. Les "Cours intégratifs", qui offraient les postes les plus intéressants, furent attribués aux maîtres chevronnés indépendamment de toute considération politique; mais l'auteur insiste sur le caractère discutable de leur adhésion au fascisme qui se justifiait souvent par un conformisme plein d'opportunisme. La réorganisation des enseignements post-primaires et professionnels entreprise par le ministre Giuseppe Belluzzo à partir de 1928 avait un double objectif. Il s'agissait de retoucher une réforme Gentile trop sélective et de répondre aux exigences 
de formation d'une main d'œuvre qualifiée ; mais l'École secondaire de formation au travail échoua, écartelée entre ses objectifs professionnels et culturels. Quant aux écoles primaires, elles furent totalement étatisées en 1932, les communes ne conservant que la charge des bâtiments et des patronages scolaires.

10 Le livre de M. D'Ascenzo retrace l'histoire de l'école primaire italienne à travers les étapes de son étatisation, et l'exemple significatif de Bologne permet de mieux comprendre les difficultés de l'application de réformes décrétées sur le plan national. On pouvait certes souhaiter une étude plus approfondie de la vie scolaire, avec une approche plus pédagogique et moins politique; mais l'ouvrage n'en représente pas moins une importante avancée dans l'historiographie de l'enseignement en Italie. 